\title{
ESTUDIOS
}

\section{Iniciativas comunitarias en materia de Responsabilidad Social Empresarial}

\section{Antonio Blanco Prieto}

Palabras clave: Responsabilidad Social Empresarial (RSE), Responsabilidad Social Corporativa (RSC), Unión Europea (UE).

Key words: Corporate Social Responsibility, European Union.

Mots clés: Responsabilité Sociale Patronale (RSP), Responsabilité Sociale Corporative (RSC), Union Européenne (UE).

\section{Introducción}

El 20 de enero de 2009 el Consejo Estatal de Responsabilidad Social de las Empresas celebró su primera reunión. Con este acto concluía un proceso iniciado en el Pleno del Senado español del 30 de mayo de 2007 con la propuesta de creación de un Consejo Estatal de Responsabilidad Social de las Empresas' , un órgano dependiente del Ministerio de Trabajo e Inmigración con carácter asesor y consultivo en materia de Responsabilidad Social Empresarial (RSE)². Para dotar de contenido y estructura

' El Consejo Estatal de Responsabilidad Social de las Empresas ha sido regulado por el Real Decreto $221 / 2008$ de 15 de febrero.

2 El Comité de Expertos en Responsabilidad Social Empresarial convocado por el Ministerio de Trabajo y Asuntos Sociales de España (2007), en su Informe de Trabajo expresa su opción por el concepto de Responsabilidad Social Empresarial (RSE) frente al concepto de Responsabilidad Social Corporativa (RSC) para evitar confusiones con los términos anglosajones "corporation" y "corporate" relativos a grandes entidades con cotización bursátil. Sin embargo, aunque se detecta un cambio progresivo del concepto de RSC por el de RSE, en el presente artículo se utilizan indistintamente ambos conceptos 
al futuro Consejo Estatal, el Ministerio consideró necesario crear un Foro de Expertos sobre el tema. En coherencia con los planteamientos precedentes de la Comisión y el Parlamento Europeo, el Informe elaborado por Foro del Expertos defiende que la Responsabilidad Social Empresarial (RSE) puede constituir un desarrollo natural y novedoso como parte del modelo social europeo. Con ello se manifiesta la naturaleza social de la RSE al tratarse de un proceso iniciado y desarrollado en la propia sociedad, que tiene como protagonistas a agentes sociales $y$, finalmente, tiene un objetivo social porque se dirige a mejorar la función social de la empresa o, dicho de otro modo, "su responsabilidad ante la sociedad".

La creación del Consejo Estatal de Responsabilidad Social de las Empresas supone una respuesta al llamamiento de la Cumbre de Lisboa a favor de una sensibilidad social de las empresas. Este planteamiento, recogido en 2001 en el Libro Verde. Fomentar un marco europeo para la responsabilidad social de las empresas, supuso el apoyo definitivo de la Comisión de las Comunidades Europeas a "un concepto con arreglo al cual las empresas deciden voluntariamente contribuir al logro de una sociedad mejor y un medio ambiente más limpio". Con la crisis de los mercados internacionales, esta contribución se hace aún más relevante y, como se ha propuesto en la primera reunión del Consejo Estatal de Responsabilidad Social de las Empresas, se considera necesario analizar en profundidad el papel de la RSE en el contexto de la actual crisis económica, al mismo tiempo que se promociona el consumo y la inversión socialmente responsable.

Estos planteamientos nos retraen al postulado característico de la economía neoclásica, recogido en el Informe Brundtland de 1987, que trata de demostrar que no hay contradicción entre sostenibilidad y desarrollo económico, sino una relación positiva y proporcional que habrá de llevarnos hacia un desarrollo sostenible, hacia una sociedad capaz de satisfacer sus necesidades sin poner en peligro las capacidades de las generaciones futuras para satisfacer sus propias necesidades (Pardo, 1997). Esta relación triangular equilibrada es consonante con los tres pilares de la Estrategia de Lisboa (a saber, el pilar económico orientado a desarrollar una economía competitiva y basada en el conocimiento; el pilar social, cuya meta es la modernización del modelo social europeo; y el pilar medioambiental, propuesto en el Consejo de Gotemburgo, que aboga por un crecimiento económico respetuoso con los recursos naturales).

por no ser evidente su diferenciación en las fuentes de información empleadas y, de acuerdo con Rodríguez (2007: 102), podríamos atribuir igual significado a los conceptos de Responsabilidad Social Empresarial (RSE), Responsabilidad Social de la Empresa (RSE), Responsabilidad Social Corporativa (RSC), Responsabilidad Corporativa o Responsabilidad Empresarial. 
Para valorar el origen conceptual y doctrinal de la RSE y su relación explícita con los pilares de la Estrategia de Lisboa, en el presente artículo se describen y relacionan los principales documentos e iniciativas de la Unión Europea en materia de sostenibilidad y Responsabilidad Social de las Empresas, desde el planteamiento expuesto en el Consejo Europeo de Lisboa en marzo de 2000 hasta la Resolución del Parlamento Europeo publicada siete años más tarde, el 13 de marzo de 2007, sobre la responsabilidad social de las empresas.

\section{Respaldo Comunitario al Desarrollo Sostenible.Los Consejos Europeos de Lisboa, Niza y Gotemburgo}

En marzo de 2000, los Jefes de Estado y de Gobierno de los países de la Unión Europea manifestaron, con la denominada Estrategia de Lisboa, su compromiso para lograr una economía dinámica y competitiva basada en el conocimiento. En concreto, los días 23 y 24 de marzo, el Consejo Europeo celebró una sesión especial en la ciudad lusa con la finalidad de establecer un nuevo objetivo estratégico de la Unión para la primera década del siglo XXI: Convertirse en la economía basada en el conocimiento más competitiva y dinámica del mundo, capaz de crecer económicamente de manera sostenible con más y mejores empleos y con mayor cohesión social. La consecución de dicho objetivo requiere: 1/ la implantación de políticas y líneas de acción dirigidas tanto al desarrollo de la sociedad de la información y de I + D como a la aceleración del proceso de reforma estructural que permita modernizar el modelo social europeo; y 2/ la aplicación de medidas macroeconómicas capaces de mantener un marco económico sólido y un crecimiento realista del $3 \%$ aproximadamente para los próximos años.

Van Lancker (2001), diputada del Parlamento Europeo por el SPA belga, recuerda que la ambición de la Cumbre de Lisboa es alcanzar la calidad social en Europa gracias al triángulo equilátero definido por la economía, el empleo y la política social. Para reforzar el papel y función de la política social en este equilibrio, la propia Cumbre solicitó la elaboración de la Agenda Social, que sería desarrollada por el Consejo de Niza celebrado en diciembre de 2000. Definida para un período inicial de 2000 a 2005, la Agenda Social concreta la política social europea a medio plazo y la involucra, por primera vez, en el más alto nivel de agenda comunitaria, algo necesario para responder a los nuevos desafíos de la sociedad del conocimiento, especialmente los derivados de los fenómenos migratorios, la implantación progresiva de las nuevas tecnologías de la información, las limitaciones de la política de empleo y protección social, y 
los nuevos riesgos de exclusión social y de pobreza en los países miembros de la Unión Europea ${ }^{3}$.

La Cumbre de Lisboa y la Agenda Social de la Cumbre de Niza, ambas en el año 2000, han permitido estructurar la Estrategia de Lisboa en tres pilares o áreas de actuación que habrían de desarrollarse en los Consejos Europeos posteriores: en primer lugar, un pilar económico orientado a desarrollar una economía competitiva, dinámica y basada en el conocimiento; en segundo lugar, un pilar social, cuya meta es la modernización del modelo social europeo, señalando como medios necesarios para lograrla la inversión en recursos humanos, la lucha contra la exclusión social y la política activa de empleo; $y$, en tercer lugar, un pilar medioambiental desde el que se plantea la necesidad de un crecimiento económico respetuoso con los recursos naturales. Este último pilar ha sido propuesto en el Consejo Europeo de Gotemburgo, de junio de 2001, defendiendo la necesidad de disociar el crecimiento económico del empleo utilitarista de los recursos naturales y alineándose de este modo la Unión Europea con el concepto de "desarrollo sostenible".

Para implantar la Estrategia de Lisboa se consideró necesario adaptar la política económica y aquellos procesos de coordinación de políticas relacionados con el empleo y el desarrollo económico. En este sentido, la Comunicación de la Comisión relativa a la racionalización de los ciclos anuales de coordinación de la política económica y de la política de empleo (COM (2002) 487, no publicada en el Diario Oficial) expone en sus antecedentes cómo, a través del Proceso de Lisboa, el Consejo Europeo habrá de definir un método abierto de coordinación de las Orientaciones Generales de Política Económica (OGPE) para el periodo 2005-2008 y de otros procesos relacionados con el empleo y el crecimiento económico, como el Proceso de Luxemburgo para el empleo, el Proceso de Cardiff sobre la reforma económica y el Proceso de Colonia, que define el diálogo macroeconómico con los agentes sociales dentro del Pacto Europeo para el Empleo4.

\footnotetext{
${ }^{3}$ La Agenda Social del Consejo de Niza establece los siguientes ejes centrales: la calidad en el empleo y mayor atención a la reglamentación social; la calidad en las relaciones laborales, con especial atención a las derivadas de procesos de reestructuración empresarial; la calidad de la política social para atajar el crecimiento de la pobreza y exclusión social; $y$, de forma transversal, tanto la promoción de la igualdad entre hombres y mujeres como el mainstreaming de la política social, recordando que su implantación debe estar integrada en todos los ámbitos de la política comunitaria de forma coherente y consistente.

${ }^{4}$ El Consejo Europeo de Lisboa solicitó la aplicación del método abierto de coordinación para que los Estados miembros pudiesen desarrollar sus propias políticas respetando la convergencia con los objetivos principales de la Unión Europea. Este método, junto con las herramientas de coordinación de políticas y procesos relacionados con empleo y crecimiento económico, no mostraron estar subordinados
} 
Como hemos indicado, con el fin de convertir a Europa en la economía más dinámica y competitiva en la escena global, a las iniciativas sociales y económicas definidas en la Estrategia de Lisboa en el año 2000, el Consejo Europeo de Gotemburgo añadiría, en 2001, las iniciativas medioambientales. Concretamente, en el Título II de sus Conclusiones se define una "Estrategia para el desarrollo sostenible". El Consejo de Gotemburgo identifica cuatro objetivos medioambientales de carácter prioritario: la lucha contra el cambio climático; la garantía de un transporte sostenible; la respuesta a las amenazas a la salud pública; y una gestión más responsable de los recursos naturales. Para definir estos objetivos, el Consejo Europeo de Gotemburgo se apoya en la Comunicación de la Comisión de 15 de mayo de 2001 sobre "Desarrollo sostenible en Europea para un mundo mejor: Estrategia de la UE para un desarrollo sostenible", en el Sexto Programa Comunitario de Acción en materia de medio ambiente, y en las Estrategias Sectoriales de Integración ambiental.

En las Conclusiones de la Presidencia del Consejo Europeo de Gotemburgo se conceptualiza el desarrollo sostenible como el afrontamiento de las necesidades de la generación actual sin poner en peligro las de las futuras generaciones. De forma específica, en la Propuesta de la Comisión ante el Consejo Europeo de Gotemburgo que define la Estrategia de la Unión Europea para un desarrollo sostenible se inicia el Título I citando el concepto aportado en 1987 en la Comisión mundial para el desarrollo del medio ambiente (Comisión Brundtland): "El desarrollo sostenible es aquel que satisface las necesidades actuales sin poner en peligro la capacidad de las generaciones futuras de satisfacer sus propias necesidades". Este fin exige utilizar como medio las políticas económicas, sociales y ambientales de forma interactiva, pues, en caso contrario, los costes para la sociedad se incrementarían, o incluso podrían hacerse irreversibles aquellas tendencias que amenazan la futura calidad de vida en los Estados de la Unión Europea, aunque sin olvidar que el desarrollo sostenible es un objetivo de alcance mundial.

Si bien Pardo (1997) considera que el significado de "desarrollo sostenible" llega a tener importantes disparidades, incluso que se trata de un concepto más ideológico y político que teórico, desde el año 2002 se revisa anualmente en los Consejos Europeos de Primavera el grado de aplicación de la Estrategia de Desarrollo

a mecanismos de coordinación eficaces, por lo que el Consejo de Barcelona manifestó la necesidad de racionalizar los diferentes procesos de coordinación para lograr unos mejores resultados. A nivel operativo, se logró simplificar el proceso inicial y las directrices para el crecimiento y el empleo se vinculan a las directrices de políticas macro y macroeconómicas, lo que sirve de criterio tanto para la Estrategia de Lisboa como para las políticas nacionales. 
Sostenible, velando por su cumplimiento y definiendo unos principios directores plasmados en la Comunicación de la Comisión relativa a la revisión de la Estrategia para un desarrollo sostenible, de 13 de diciembre de 2005. En la Plataforma de Acción, recogida en esta Comunicación al Consejo y al Parlamento Europeo, se añaden a los cuatro objetivos medioambientales del Consejo de Gotemburgo dos nuevos objetivos: 1/ inclusión social, demografía y flujos migratorios; y 2/ pobreza mundial y retos en materia de desarrollo. De forma transversal a los seis objetivos o ámbitos principales de trabajo, la gestión de la producción y el consumo sostenible se presentan prioritarios y directamente relacionados con la Responsabilidad Social Empresarial (RSE). No en vano esta Comunicación recuerda que el reto de reforzar recíprocamente el crecimiento económico, el bienestar social y la protección del medio ambiente, no se ciñe exclusivamente a la actuación pública. Aunque se reconozca que las autoridades públicas constituyen una parte importante de la solución al problema, la Comisión considera necesario facultar y alentar a todas las partes, en especial a los ciudadanos y a las empresas, para que sean partícipes del cambio, aportando ideas, aprovechando oportunidades y respondiendo al reto de un desarrollo sostenible.

Este planteamiento no debe interpretarse como una nueva carga para el sector empresarial, sino como fuente de nuevas oportunidades. En concreto, la Plataforma de Acción para el cumplimiento de la Estrategia para un desarrollo sostenible nos recuerda que "cada vez más dirigentes empresariales reconocen los beneficios de ocuparse de los aspectos relacionados con el desarrollo sostenible" ${ }^{2}$, pues las empresas más exitosas son conscientes de la necesidad de planificar las inversiones a largo plazo en nuevas tecnologías y con métodos demandados por los consumidores, aunque también necesitan un marco reglamentario que facilite $o$ incentive una actuación estable en esta dirección. Por ello, entre las iniciativas propuestas por la Comisión se urge a los dirigentes empresariales a iniciar una reflexión junto con los responsables políticos para proponer respuestas ambiciosas que superen los mínimos establecidos por las legislaciones vigentes, fomentando la responsabilidad social de las empresas y complementando el diálogo con la sociedad civil. De forma más específica, entre los diez principios rectores de las políticas para un desarrollo sostenible, un principio hace referencia explícita a la participación de las empresas y de los interlocutores sociales, aumentando la responsabilidad social de las empresas y fomentado las asociaciones entre el sector público y el privado para conseguir una producción y consumo sostenibles.

${ }^{5}$ La Plataforma de Acción queda recogida en la Comunicación de la Comisión relativa a la revisión de la Estrategia para un desarrollo sostenible, p. 16. 


\section{Libro Verde}

En coherencia con la estrategia de desarrollo sostenible para Europa acordada en el Consejo de Gotemburgo de junio de 2001, la Comisión de la Unión Europea presentó un mes más tarde un documento para fomentar un marco europeo para la responsabilidad social de las empresas, conocido como el Libro Verde. Este documento nace con el propósito de transformar la economía europea en la más competitiva del entorno global, capaz de generar, no sólo riqueza económica, sino también bienestar social y sostenibilidad medioambiental, siguiendo el llamamiento del Consejo Europeo de Lisboa de marzo de 2000. La Unión Europea muestra de esta forma su interés por la RSE, aportando información para el fomento y desarrollo de prácticas innovadoras y proponiendo un modelo de trabajo basado en asociaciones profundas en las que todos los agentes desempeñen un papel activo. Siguiendo la llamada efectuada en 1993 por el Presidente de la Unión Europea, Jacques Delors, el Libro Verde respalda las iniciativas empresariales de fomento de la responsabilidad social y promueve un cambio de impresiones a favor de nuevas actuaciones, por lo cual se define el objeto de iniciar un debate sobre la forma más adecuada para que la Unión Europea fomente la RSE tanto a nivel europeo como internacional, aprovechando al máximo las prácticas existentes, potenciado el desarrollo de nuevas experiencias, e incrementando la transparencia, fiabilidad de la evaluación y la validación de la RSE.

El Libro Verde incluye en su introducción la definición de "Responsabilidad Social de las Empresas" (RSE), entendiendo por tal, esencialmente, un concepto con arreglo al cual las empresas deciden voluntariamente contribuir al logro de una sociedad mejor y un medio ambiente más limpio. La definición aporta dos aspectos clave: el carácter voluntario de la RSE y su finalidad de desarrollo sostenible. El carácter no obligatorio no exime del cumplimiento de la legislación existente en materia social, laboral o medioambiental, pues la RSE no es sustitutiva de ninguna reglamentación o legislación, ni tampoco implica el simple cumplimiento de las obligaciones jurídicas, sino que supone ir más allá de las mismas. Con ello las empresas son partícipes del objetivo del Consejo Europeo de Lisboa de desarrollar una economía basada en el conocimiento, competitiva, dinámica y, a su vez, socialmente cohesionada, así como son partícipes también de la Agenda Social Europea aprobada en el Consejo de Niza al vincularse también la RSE con las políticas laborales y sociales de la nueva economía. A nivel internacional, el Libro Verde reconoce la importancia del hecho que el enfoque europeo de RSE refleje y esté integrado en el contexto internacional en el que se inscribe el Global Compact de Naciones Unidas, la Declaración tripartita de la OIT sobre empresas multinacionales y política social, y las Líneas Directrices de la OCDE. Conscientes de la existencia e importancia de 
estas iniciativas, la contribución de un enfoque europeo de RSE estará centrada en establecer un marco global europeo para elaborar principios, instrumentos operativos y promoción de la calidad y la coherencia de las prácticas existentes así como de ideas innovadoras.

Respecto a las prácticas susceptibles de ser ejecutadas y evaluadas, éstas hacen referencia a diferentes áreas de gestión, pues responden a las expectativas de los diferentes públicos de la organización. Concretamente el Libro Verde señala las diferentes áreas, objetivos y prácticas propias de la RSE, todo ello clasificado según afecte a la dimensión interna o externa de la organización. Además de estos aspectos, el Libro Verde recuerda la necesidad de adoptar un modelo de gestión integrada de la RSE, por lo cual las empresas deben, en un primer momento, adoptar una declaración de principios o manifiesto en el cual se reflejen los valores de la organización y el compromiso de sus máximos niveles directivos. Por ello se considera necesario incrementar los esfuerzos en formación de directivos y trabajadores para que comprendan el alcance y procedimientos de implantación de la responsabilidad social en sus empresas. Así mismo, una vez desarrollada la gestión, el documento de la Comisión apoya la elaboración de informes sociales y medioambientales que reflejen las prácticas y faciliten la transparencia informativa gracias a la comprobación de los datos publicados por parte de terceros independientes. Para una elaboración de informes aplicables en cualquier medio cultural y país se hace referencia a las normas internacionales como los convenios identificados en la Declaración de 1998 de la OIT, aunque también se cita Guía de la Global Reporting Initiative (modelo a seguir en el ámbito del medio ambiente) y a otra iniciativa como la norma Social Accountability 8000 (SA 8000), cuyo sistema de control está basado en auditorías de calidad como las empleadas con la norma ISO 9000.

\section{El seguimiento comunitario del Libro Verde}

El 3 de diciembre de 2001, el Consejo de la Unión Europea habría de realizar una Resolución relativa al seguimiento del Libro Verde sobre la responsabilidad social de las empresas (2002/C 86/03), reconociendo explícitamente que la RSE puede contribuir al logro de los objetivos establecidos por los Consejos Europeos de Lisboa, Niza y Gotemburgo y, a su vez, puede ser capaz de fomentar la integración social y el desarrollo sostenible. En este sentido el Consejo reconoce la importancia de la RSE como medio para lograr este fin, pues se trata de un instrumento que podría contribuir a fomentar un alto nivel de cohesión social, protección medioambiental 
y respeto de los derechos fundamentales, al mismo tiempo que se mejora la competitividad de todos los tipos de empresa y en cualquier sector de actividad. Por ello, completando las normativas y legislación en materia de derechos sociales o medioambientales, la RSE en general y los códigos de conducta empresarial en particular pueden promover una actitud responsable de las empresas que aporta valor añadido a las acciones existentes a nivel local y nacional. Concretamente, el Consejo considera útil fomentar el intercambio de buenas prácticas e ideas innovadoras en materia de RSE, una mayor investigación y divulgación de la relación existente entre prácticas socialmente responsables y resultados económicos de las empresas, así como las iniciativas dirigidas a la formación de directivos y trabajadores sobre el tema para que puedan integrar criterios de RSE en la planificación estratégica y operaciones cotidianas.

Por los motivos previamente descritos, el Consejo, en su Resolución de 3 de diciembre de 2001, conviene acoger favorablemente el seguimiento y cumplimiento del Libro Verde para "fomentar un marco europeo para la responsabilidad social de las empresas" y solicita a la Comisión la integración en una próxima Comunicación de los debates llevados a cabo por los Estados miembros en el periodo de consulta. Esta Comunicación, relativa a la responsabilidad social de las empresas y a la contribución empresarial al desarrollo sostenible, habría de realizarse el 2 de julio de 2002 (no publicada en el Diario Oficial de la Unión Europea) y dejaría constancia de las respuestas recibidas por la Comisión a la consulta abierta sobre el Libro Verde. En total se recibieron más de 250 respuestas $^{6}$, de procedencia heterogénea, al proponerse dictámenes tanto de instituciones europeas (Consejo, Parlamento, Comité Económico y Social, Comité de las Regiones), como de Estados miembros, como de organizaciones patronales, empresas, agentes sociales, asociaciones y entidades de la sociedad civil, representantes del mundo académico y personas interesadas. Como norma general, aunque se aprecia la satisfacción con el Libro Verde y la conformidad con un debate abierto sobre el concepto de RSE, se recogen también divergencias atendiendo a los diversos tipos de organizaciones que emitieron respuestas, tal como se puede observar en la tabla 1:

\footnotetext{
${ }^{6}$ Todas las respuestas al Libro Verde así como la identificación de las organizaciones que las proponen puede encontrarse en [http://ec.europa.eu/employment_social/soc-dial/csr/csr_responses.htm]. Acceso el día 2 de julio de 2008.
} 
TABLA I. Resumen de la consulta sobre el Libro Verde relativo a la RSE según participantes ${ }^{7}$

\begin{tabular}{|l|l|}
\hline \multicolumn{1}{|c|}{ Entidad } & \multicolumn{1}{c|}{ Propuesta } \\
\hline Earlamento & $\begin{array}{l}\text { Integración del concepto de RSE en todos los ámbitos de com- } \\
\text { petencia de la Unión Europea, especialmente en la financiación } \\
\text { de medidas sociales y regionales. } \\
\text { Establecimiento de una plataforma multilateral sobre RSE. } \\
\text { Propuesta de inclusión en las memorias de las empresas de } \\
\text { un triple balance con indicadores de resultados económicos, } \\
\text { sociales y medioambientales, incluida la dimensión de dere- } \\
\text { chos humanos. }\end{array}$ \\
\hline $\begin{array}{l}\text { Comité de las } \\
\text { Regiones }\end{array}$ & $\begin{array}{l}\text { Propuesta de proporcionar a nivel europeo un marco de ac- } \\
\text { ción que permita aumentar la sensibilización, los principios } \\
\text { responsables yla ayuda a las empresasy autoridades públicas } \\
\text { para integrar en sus actividades la responsabilidad social. }\end{array}$ \\
\hline $\begin{array}{l}\text { Comité } \\
\text { Económico y }\end{array}$ & $\begin{array}{l}\text { Propuesta de que las nuevas iniciativas europeas en el ámbito } \\
\text { de la RSE sean coherentes con los acuerdos suscritos en el } \\
\text { ámbito de las organizaciones internacionales. Del mismo } \\
\text { modo, habrán de respetar tanto la acción voluntaria como } \\
\text { los principios de sostenibilidad medioambiental, económica } \\
\text { y social. }\end{array}$ \\
\hline $\begin{array}{l}\text { Consejo de la } \\
\text { Unión Europea }\end{array}$ & $\begin{array}{l}\text { Indicación de que el enfoque europeo de RSE pueda servir } \\
\text { para desarrollar su concepto y para completar las medidas } \\
\text { existentes a nivel local y nacional. } \\
\text { Confianza en la contribución de la RSE en la mejora de la } \\
\text { competitividad empresarial y en el fomento de un elevado } \\
\text { nivel de cohesión social, de protección del medio ambiente y } \\
\text { de respeto de los derechos humanos. } \\
\text { (Resolución de 3 de diciembre de 2001) }\end{array}$ \\
\hline
\end{tabular}

7 Fuente: Comunicación de la Comisión relativa a la responsabilidad social de las empresas: una contribución empresarial al desarrollo sostenible (COM (2002) 347 final, no publicada en el Diario Oficial). 


\begin{tabular}{|l|l|}
\hline $\begin{array}{l}\text { Organizaciones } \\
\text { de consumidores }\end{array}$ & $\begin{array}{l}\text { Recordatorio de la importancia de ofrecer información sobre } \\
\text { las condiciones éticas, sociales y ecológicas de producción y } \\
\text { comercialización de bienes y servicios. Esta información habrá } \\
\text { de ser fiable y exhaustiva para orientar a los consumidores } \\
\text { en sus decisiones de compra. }\end{array}$ \\
\hline Inversores & $\begin{array}{l}\text { Propuesta de mejora de la divulgación de la información y la } \\
\text { transparencia de las prácticas empresariales, así como de la } \\
\text { metodología utilizada por las agencias de calificación y por } \\
\text { la gestión de fondos de inversión socialmente responsable } \\
\text { (ISR) y de fondos de pensiones. }\end{array}$ \\
\hline $\begin{array}{l}\text { Sindicatos y } \\
\text { organizaciones } \\
\text { de la sociedad } \\
\text { civil }\end{array}$ & $\begin{array}{l}\text { Desarrollode un marco reglamentario con normas mínimas para } \\
\text { garantizar unas reglas equitativas en materia de RSE, conside- } \\
\text { rando que las iniciativas voluntarias no son suficientes para sal- } \\
\text { vaguardar los derechos de los trabajadores y los ciudadanos. } \\
\text { Definición, aplicación y evaluación de las prácticas de RSE } \\
\text { mediante la participación de todas las partes interesadas y } \\
\text { no de forma unilateral por las propias empresas. }\end{array}$ \\
$\begin{array}{l}\text { Introducción de mecanismos eficaces que obliguen a las } \\
\text { empresas a responsabilizarse de las consecuencias sociales } \\
\text { y medioambientales de sus actividades. }\end{array}$ \\
\hline $\begin{array}{l}\text { Organizaciones } \\
\text { empresariales }\end{array}$ & $\begin{array}{l}\text { Defensa de la naturaleza voluntaria de la responsabilidad } \\
\text { social, su integración en el contexto del desarrollo sostenible } \\
\text { y la necesidad de definir su contenido a escala global. } \\
\text { Se considera contraproducente cualquier solución con pre- } \\
\text { tensión de universalidad o cualquier propuesta reguladora a } \\
\text { nivel europeo, al anular el carácter innovador de las empresas } \\
\text { y generar posibles conflictos de prioridades para aquellas } \\
\text { empresas que operan en diferentes áreas geográficas. }\end{array}$ \\
\hline
\end{tabular}

En la citada Comunicación relativa a la responsabilidad social de las empresas, de julio de 2002, se propone una estrategia de promoción de la RSE respaldada en seis principios de acción comunitaria: el reconocimiento de la naturaleza voluntaria de la RSE (tal como había sido también propuesto por las organizaciones empresariales en el proceso de consulta sobre el Libro Verde); el desarrollo de prácticas de responsabilidad social creíbles y transparentes; la focalización en aquellas actividades en las que la intervención de la Comunidad pueda aportar 
valor añadido; la inclusión equilibrada de las dimensiones económica, social y ecológica en el enfoque global de RSE, sin olvidar los intereses de los colectivos de consumidores; apoyo y atención a las necesidades y características específicas de las PYMES; y coherencia con los acuerdos internacionales existentes en materia de RSE (concretamente las normas fundamentales del trabajo de la OIT y las directrices de la OCDE para empresas multinacionales).

Para el cumplimiento de estos principios, la Comisión propone centrar su estrategia en una mayor difusión internacional-especialmente en los países en desarrollo-del impacto positivo de la RSE tanto en la sociedad como en las empresas, así como promover una mejora en las capacidades de gestión sobre la materia, facilitar la transparencia informativa y fomentar el intercambio de buenas prácticas entre las empresas, prestando una especial atención a la implantación en las PYME. Del mismo modo, se propone integrar la responsabilidad social en las políticas comunitarias y fomentar la creación en la Unión Europea del Foro Multilateral con la participación de todas las partes implicadas para garantizar la aceptación y credibilidad de la RSE.

\section{Foro Multilateral Europeo}

En el año 2002, se puso en marcha el Foro Multilateral Europeo sobre RSE, formado por una plenaria con representantes de las partes interesadas, que se reúnen dos veces al año. El Foro concedió gran importancia al diálogo entre las partes y se incentivaron sus participaciones con reuniones más frecuentes en mesas redondas temáticas. Bajo la presidencia de la Comisión, el Foro propuesto en la Comunicación de la Comisión relativa a la responsabilidad social de las empresas reunió a una representación equilibrada de los empresarios, a la representación de los trabajadores a través de los sindicatos, a los consumidores, la sociedad civil, las asociaciones profesionales y las redes empresariales, siendo invitadas las otras instituciones de la Unión Europea en calidad de observadoras y desempeñando la Comisión un rol dinamizador. Tal como recuerda Sagardoy (2007), entre los principales objetivos del Foro destacaba el que perseguía mejorar el conocimiento sobre la relación existente entre el desarrollo sostenible y la RSE, empleando para ello el intercambio de experiencias y buenas prácticas, así como valorar la idoneidad de definir principios comunes para las prácticas de responsabilidad corporativa partiendo para ello de las iniciativas existentes en la Unión Europea y la legislación vigente.

En la Resolución del Consejo de 6 de febrero de 2003 relativa a la responsabilidad social de las empresas (2003/C 39/02), el Consejo insta a la Comisión y a las 
organizaciones representadas en el Foro Multilateral a que continúen "haciendo hincapié en la forma en que la RSE puede contribuir a los objetivos de la Unión Europea, establecidos en particular por los Consejos Europeos de Lisboa, Niza y Gotemburgo". Para ello, también se recuerda la necesidad de velar por el respeto e inclusión de los puntos de vista de todos los agentes interesados a escala europea, nacional, regional y local, así como la transparencia y eficiencia en los trabajos realizados en el Foro. En el año 2004, el Foro presentó su informe final y, aunque se reconoció la necesidad de fijar un conjunto de principios o guías para la implantación de la RSE en la Unión Europea, se manifestaron divergencias sobre la forma y alcance de dichas normas, centrándose especialmente el debate sobre la posible necesidad de contar con una normativa comunitaria en materia de RSE y sobre la obligatoriedad de las empresas de informar a sus públicos sobre sus iniciativas de responsabilidad social. Aquellos puntos en los que destacó el consenso son los relacionados con la necesidad de profundizar el conocimiento teórico y la investigación sobre la responsabilidad social de las empresas, así como con la necesidad de continuar realizando campañas de sensibilización y desarrollando competencias de gestión y trabajo para integrar criterios sociales y medioambientales en las líneas estratégicas de las empresas, valorando para ello el acuerdo de los diferentes públicos o stakeholders de las mismas.

Como consecuencia del Foro de RSE y de los debates planteados en él, se ha logrado una mejoría en la sensibilización, comprensión y aceptación de la responsabilidad social de las empresas, concluyendo que si los agentes implicados aplicasen las recomendaciones alcanzadas íntegramente, se lograría un avance significativo de la RSE en Europa y en el mundo. Del mismo modo, el Foro alumbraría el nacimiento de una interpretación común europea sobre el significado de la RSE: integración voluntaria, por parte de las empresas, de las preocupaciones sociales y medioambientales en sus operaciones comerciales y sus relaciones con sus interlocutores ${ }^{8}$. Al igual que en el Libro Verde, el Foro de RSE subraya el carácter voluntario de la responsabilidad social en la contribución de las empresas a una sociedad mejor y un medioambiente más limpio, aunque se añade que esta contribución está relacionada con sus operaciones comerciales y con las relaciones que las empresas establecen con sus interlocutores o públicos objetivo, lo cual supera el mero mecenazgo o filantropía empresarial para situarse en el marco estratégico de gestión.

${ }^{8}$ Esta definición queda recogida en la Comunicación de la Comisión al Parlamento Europeo, al Consejo y al Comité Económico y Social Europeo, 22 de marzo de 2006. Poner en práctica la asociación para el crecimiento y el empleo: Hacer de Europa un polo de excelencia de la Responsabilidad Social de las Empresas, p. 5. 


\section{Un marco europeo para la responsabilidad social de las empresas}

Reconociendo que la globalización suponeoportunidades, pero también complejidad organizativa para las empresas, y que por ello un planteamiento equilibrado de la RSE debe incluir tanto sus aspectos económicos, sociales y medioambientales como los intereses de los consumidores, la Resolución del Consejo de la Unión Europea de 6 de febrero de 2003 relativa a la responsabilidad social de las empresas (2003/C 93/02) recuerda las intenciones de la Comisión de fomentar un marco europeo para la misma, instando para ello a los Estados miembros a trabajar de forma paralela al desarrollo de una estrategia comunitaria, procurando que sus empresas sean conscientes de sus beneficios y de los resultados de una cooperación entre gobiernos, empresas y otros sectores de la sociedad. Por ello se recomienda la incorporación de la RSE en sus políticas nacionales al mismo tiempo que fomentan la transparencia de las prácticas e instrumentos de RSE.

Siguiendo este planteamiento, el Parlamento Europeo, vistas las Resoluciones, Comunicaciones y documentaciones precedentes en materia de RSE y de desarrollo sostenible, dicta una Resolución sobre la Comunicación de la Comisión relativa a la responsabilidad social de las empresas, recordando la contribución empresarial al desarrollo sostenible. Se considera, entre otras cuestiones, que el concepto de RSE es relevante para todo tipo de empresa, con independencia de sus dimensiones, pues a las empresas incumbe una función social y la responsabilidad con todas las partes implicadas en la actividad empresarial, reconociendo que el concepto engloba diversas facetas y diferentes elementos de prácticas responsables desde el punto de vista social y ambiental. Dicha Resolución respalda el deseo de la Comisión de garantizar el desarrollo de los principios y políticas de RSE a escala comunitaria tras la finalización de los dos años iniciales de trabajos del Foro multilateral, solicitando el consenso entre sus miembros para emprender acciones futuras de carácter conjunto. Todo ello con la finalidad de desempeñar una contribución clave al desarrollo sostenible y, al mismo tiempo, de reforzar el potencial innovador y la competitividad de Europa, tal como reconoció la Comisión en la Comunicación al Consejo Europeo de Primavera de marzo de 2005.

Se define el objetivo de constituir una Europa social en la economía mundial, lo que constituye el lema de la segunda fase de la Agenda Social vigente hasta 2010. Para ello se recuerda la necesidad de obrar a favor del desarrollo sostenible de Europa gracias al crecimiento económico equilibrado y la estabilidad de los precios en una economía social de mercado, capaz de conjugar la competitividad con el pleno empleo, el progreso social y un nivel elevado de protección y mejora 
de la calidad del medio ambiente. Desde un punto de vista más operativo para la fase 2006-2010, la Agenda Social define dos ejes prioritarios: lograr el pleno empleo y establecer una nueva dinámica para las relaciones laborales. Para ello, entre otras acciones públicas, la Comisión reitera su compromiso de fomento de la responsabilidad social de las empresas en cooperación con los Estados miembros y sus protagonistas, presentando iniciativas para mejorar más aún el desarrollo y transparencia de la RSE. Por ello, la Comisión anunció en la Agenda Social la presentación de iniciativas de cooperación con los Estados miembros y las partes interesadas con la finalidad de mejorar progresivamente el desarrollo y la transparencia de la RSE. Este objetivo recuerda la importancia de que los dirigentes empresariales y demás partes interesadas en Europa inicien, junto con los responsables políiticos, una reflexión sobre las políticas más adecuadas para un desarrollo sostenible, proponiendo también respuestas empresariales que superen la legislación y normas mínimas vigentes, tal como urge la Comisión en la Comisión relativa a la revisión de la Estrategia para un desarrollo sostenible de diciembre de 2005.

\section{Un polo de excelencia de la responsabilidad social de las empresas}

La Comunicación de la Comisión al Parlamento Europeo, al Consejo y al Comité Económico y Social Europeo, de 22 de marzo de 2006, lleva por título "Poner en práctica la asociación para el crecimiento y el empleo: Hacer de Europa un polo de excelencia de la Responsabilidad Social de las Empresas". En ella, se vuelve a recordar el carácter voluntario por parte de las empresas de la RSE, valorando el papel activo que las organizaciones empresariales pueden tener en la conciliación de las ambiciones económicas, sociales y ambientales de la Unión Europea. Tras el reconocimiento de que la economía de mercado prevalece en la escena global posterior a la guerra fría y de la importancia de ofrecer nuevas oportunidades para las empresas, también se manifiesta la necesidad de fijar límites y movilizarse a favor del bienestar social de las democracias modernas. Para ello, las empresas no sólo podrían decidir superar los requisitos jurídicos y normativas mínimas recogidas en las negociaciones colectivas y abordar necesidades de la sociedad de diversa índole, sino también mostrar su protagonismo y colaborar con el desarrollo de la RSE gracias a la aportación de contribuciones y experiencias positivas.

Aunque las prácticas de RSE no podrían alcanzar resultados por sí mismas ni ser sustitutivas de la función pública, la relación positiva entre acciones de RSE y 
política queda plasmada en la citada Comunicación a la Comisión (COM (2006) 136 final) al hacer referencia diferentes objetivos políticos, tal como se muestra en la tabla 2:

TABLA 2

\begin{tabular}{|l|l|}
\hline \multicolumn{1}{|c|}{ Prácticas de RSE } & \multicolumn{1}{|c|}{ Objetivos políticos de la UE } \\
\hline $\begin{array}{l}\text { Contratación de más trabajadores de } \\
\text { grupos desfavorecidos. }\end{array}$ & $\begin{array}{l}\text { Mercados laborales más integrados y } \\
\text { mayores niveles de inclusión social. }\end{array}$ \\
\hline $\begin{array}{l}\text { Desarrollo de competencias, aprendizaje } \\
\text { permanente y empleabilidad de los } \\
\text { trabajadores. }\end{array}$ & $\begin{array}{l}\text { Mantenimiento de la competitividad en } \\
\text { la economía global del conocimiento. } \\
\text { Afrontar el envejecimiento de la po- } \\
\text { blación activa. }\end{array}$ \\
\hline $\begin{array}{l}\text { Seguridadalimentaria, comercialización, } \\
\text { etiquetado responsable de productos } \\
\text { alimenticios y sustancias químicas no } \\
\text { tóxicas }\end{array}$ & Mejora en la salud pública. \\
\hline $\begin{array}{l}\text { Interacción más intensa con las partes in- } \\
\text { teresadas. Creación de entornos laborales } \\
\text { más estimulantes para la innovación. }\end{array}$ & $\begin{array}{l}\text { Rendimiento de la innovación, especial- } \\
\text { mente aquella que aborda problemas } \\
\text { sociales. }\end{array}$ \\
\hline $\begin{array}{l}\text { Inversiones en ecoinnovacióny adopción } \\
\text { voluntaria de sistemas de gestión medio- } \\
\text { ambiental y de etiquetado. }\end{array}$ & $\begin{array}{l}\text { Empleo más racional de los recur- } \\
\text { sos naturales. Mejores niveles de } \\
\text { contaminación. }\end{array}$ \\
\hline $\begin{array}{l}\text { Gestión de la comunicación e imagen } \\
\text { más favorable de las empresas y el em- } \\
\text { presariado en la sociedad. }\end{array}$ & $\begin{array}{l}\text { Generación de actitudes más favora- } \\
\text { bles hacia el espíritu empresarial. }\end{array}$ \\
\hline $\begin{array}{l}\text { Cumplimiento de normas laborales y y } \\
\text { ambientales básicas. Respecto a los } \\
\text { derechos humanos, especialmente en } \\
\text { países en vías de desarrollo. }\end{array}$ & $\begin{array}{l}\text { Respeto a los derechos humanos, pro- } \\
\text { tección medioambientaly cumplimiento } \\
\text { laboral. }\end{array}$ \\
\hline $\begin{array}{l}\text { Desarrollo e implantación de políticas a } \\
\text { medio y largo plazo necesarias para un } \\
\text { desarrollo sostenible. }\end{array}$ & $\begin{array}{l}\text { Reducción de la pobreza y avance } \\
\text { hacia los Objetivos de Desarrollo del } \\
\text { Milenio. }\end{array}$ \\
\hline
\end{tabular}

Fuente: Adaptación de la Comunicación de la Comisión (COM (2006) 136 final). 
A pesar del protagonismo indudable del sector empresarial en el desarrollo y puesta en práctica de la RSE, la Comisión reconoce la importancia de apoyar la extensión de las acciones de RSE, y se compromete a caminar en esta dirección. Para ello, plantea la asociación con las empresas y con los interesados pertinentes para aumentar la sensibilización e intercambio de las mejores prácticas a medida que la RSE siga desarrollándose, haciendo hincapié en las PYME, en aquellos Estados miembros donde la responsabilidad empresarial no haya alcanzado una amplia difusión, así como en los países adherentes y candidatos. La Comisión también expresa su apoyo a iniciativas multilaterales para estimular una mayor sensibilización y reforzar la credibilidad del concepto de RSE, así como la cooperación con los Estados miembros y los países adherentes, en especial mediante el grupo de representantes nacionales de alto nivel sobre RSE. El texto de la Comisión también destaca como medida trasladar una función socialmente responsable a los consumidores en sus decisiones de compra, para lo cual deberán disponer de información transparente sobre las características de los productos, el rendimiento social y ambiental de las empresas y la cadena de suministros hasta su origen. Todos estos aspectos deberán complementarse con la investigación interdisciplinaria sobre RSE, especialmente sobre el análisis de relaciones a nivel "macro" $\mathrm{y}$ "meso" entre RSE, competitividad y desarrollo sostenible, así como con la educación de próximas generaciones de empresarios y trabajadores sensibilizados y cualificados para la implantación de prácticas responsables en sus organizaciones. Con todo ello, se estará contribuyendo a la consecución de los Objetivos del Milenio de las Naciones Unidades y de otras declaraciones o directrices de dimensión internacional, lo cual permite a la Comisión asumir el liderazgo en la promoción global del desarrollo sostenible.

Ante estos planteamientos, la Comisión expresa su apoyo a la creación de una Alianza Europea para la RSE, abierta a las empresas europeas con independencia de su tamaño y sector, y constituida como instrumento o proceso político que permita incrementar la práctica de la responsabilidad social en el empresariado europeo. Dicha Alianza Europea será creada según la descripción anexa a la Comunicación para "Hacer de Europa un polo de excelencia de la Responsabilidad Social de las Empresas" (COM (2006) 136 final). Supondrá una cobertura política para las iniciativas de RSE de grandes empresas, PYME y sus interlocutores, y por tanto estará abierta a todo tipo de organizaciones que la apoyen de forma voluntaria. Dicho apoyo no tendrá que estar firmado en ningún acuerdo o convenio de cooperación con la Comisión ni con cualquier otra autoridad pública. Más bien supone un compromiso de las empresas con el desarrollo de los valores y principios europeos y con la ambición de hacer de Europa un polo de excelencia en materia de RSE. Se trata una asociación entre empresas que, recogiendo las lecciones del Foro 
multilateral, confíen en la capacidad de la RSE para aunar desarrollo sostenible con competitividad en los mercados.

La Alianza clasifica su actividad en tres áreas de actuación: la sensibilización sobre RSE; la integración de la RSE y el desarrollo de coaliciones de cooperación; y la garantía de un entorno favorable para la RSE. Para aumentar la sensibilización y mejorar su conocimiento se estudiarán formas novedosas de intercambio y difusión de buenas prácticas e instrumentos para la gestión de la RSE, y para ello se ofrece esta información tanto a profesionales del ámbito empresarial como a responsables políticos, consumidores, inversores y al público en general. Una buena educación en la materia supondrá una importante contribución a la futura sostenibilidad de Europa. Desde el área de trabajo de integración de la RSE y de cooperación se crean "coaliciones abiertas de cooperación" para abordar, en reuniones de estudio y de generación de proyectos operativos, temas como la innovación en tecnologías sostenibles, el crecimiento de las pyme, la integración de consideraciones sociales y ambientales en las operaciones empresariales, la consolidación del compromiso entre las partes implicadas o la operativa social y ambientalmente responsable más allá de las fronteras comunitarias. En la tercera línea de trabajo de la Alianza, la Comisión Europea y los Estados miembros han mostrado su compromiso para garantizar un entorno favorable para la RSE, fomentando esfuerzos voluntarios e innovadores de las empresas, promoviendo buenas prácticas e integrando el fomento de la RSE en las políticas oportunas.

Además del apoyo específico de la Comisión, a través de la Alianza, las empresas y las redes empresariales han presentado un documento breve de trabajo $u$ hoja de ruta ("European Roadmap for Business on CSR") que ofrece su visión, compromiso, objetivos y estrategias para su logro, al mismo tiempo que se hace un llamamiento a las empresas para que respalden esta hoja de ruta, así como a las partes interesadas para que apoyen activamente los esfuerzos de aquellas empresas que tratan de ser más competitivas y sostenibles. Este apoyo también es solicitado a la Unión Europea y a los Gobiernos, a quienes también se insta a cambiar la Estrategia de Lisboa de compromisos a resultados, poniendo en práctica políticas que hagan una Europa atractiva para la inversión y más flexible para crear más y mejores empleos, una Europa cuyo crecimiento esté basado en capacidades de alto nivel e innovación, así como políticas líderes en desarrollo sostenible y coherentes en materia de comercio y desarrollo.

En marzo de 2007, la Resolución del Parlamento Europeo "sobre la responsabilidad social de las empresas: una nueva asociación", considera que las empresas no deben sustituir a los poderes públicos en el control del respeto a las normas 
sociales y medioambientales, pero al mismo tiempo manifiesta su convicción de que una mayor responsabilidad social y medioambiental de las empresas representa un elemento esencial del Modelo Social Europeo y de la Estrategia Europea de Desarrollo Sostenible, respondiendo a su vez a los nuevos retos sociales vinculados a la globalización económica. En este sentido, se recuerda la contribución de Europa a la RSE global, pues el impacto potencial de las políticas empresariales socialmente responsables continúa siendo mayor en relación con las cadenas mundiales de proveedores, lo que ayuda a combatir la pobreza en países en desarrollo, así como a fomentar condiciones dignas de trabajo, apoyar los principios de comercio justo y buena gobernanza y favorecer el cumplimiento de normas internacionales, especialmente las normas de trabajo dictadas por la OIT en aquellos países donde la normativa nacional es laxa o inexistente. Con esta Resolución, de 13 de marzo de 2007, se cuestiona la idea de "ir más allá en el cumplimiento" de las obligaciones jurídicas en el ámbito global, pues una organización puede probar su responsabilidad social en la Unión Europea pero violar la legislación local de otros países en los que desarrolle su actividad. Por ello, el compromiso de la Unión Europea con la RSE deberá incorporar también la ayuda a gobiernos de terceros países para cumplir la legislación social y medioambiental conforme a los convenios internacionales.

Otro elemento que incorpora al debate la Resolución de 13 de marzo de 2007 es la dispersión del concepto de voluntariedad, pues, aunque la diversidad de prácticas en materia de RSE puede suponer una inspiración y motivación para las empresas, en realidad la diversidad de iniciativas voluntarias también podría considerarse un obstáculo para que un mayor número de empresas adopten políticas de RSE, así como un desincentivo de cara a las empresas para que lleven a cabo acciones de RSE más creíbles o más ambiciosas. Por ello se vuelve a defender la difusión de las buenas prácticas en materia de RSE, y se considera oportuno que la Comisión exponga el listado de criterios que las empresas deberían respetar si desean afirmar que son responsables.

\section{Conclusiones}

Desde el Libro Verde hasta la Resolución del Parlamento Europeo, de 13 de marzo de 2007, sobre la responsabilidad social de las empresas, en toda doctrina y documentación comunitaria referente al tema se aprecia el mantenimiento de una misma línea conceptual (véase Anexo I). En todo momento se hace constancia del carácter voluntario de la RSE, lo que supone la aplicación de iniciativas prácticas 
por parte de las empresas más allá de la legislación y normativas vigentes en las diferentes áreas a las que hace referencia su responsabilidad social y ambiental. Estas prácticas no son entendidas como acciones autónomas e independientes, sino que deben formar parte de una gestión planificada e integrada en la gestión estratégica de la empresa. De este modo, la RSE se inserta en el núcleo de gestión de las organizaciones para diseñar una estrategia triangular y equilibrada entre los polos económico, social y ambiental. En este sentido, se valora positivamente la integración de dicha gestión con el cumplimiento de normativas y directrices de carácter internacional, como las emanadas de la OIT, las Líneas Directrices de la OCDE o el Pacto Mundial de Naciones Unidas, lo cual no debe llevarnos a plantear que la RSE está orientada exclusivamente hacia las empresas multinacionales, sino a todo tipo de empresa, independientemente de su dimensión y sector de actividad. Por ello la Unión Europea recuerda en diferentes textos la importancia de extender la conceptualización de RSE hacia las PYME, valorando especialmente que su presencia en el tejido económico europeo es superior al $90 \%$ de las empresas. Además, las PYME pueden gestionar de forma autónoma su responsabilidad social - estar vinculadas en cadenas de suministro a grandes empresas que establecen criterios de RSE a todos los miembros de la cadena productiva. Esta cuestión también afecta a su extensión geográfica, pues la responsabilidad social de las empresas no se circunscribe, por lo general, a sus actividades dentro de un solo país, ni existen soluciones a diversas decisiones empresariales que tengan como marco a un solo Estado, sino que la globalización facilita los intercambios comerciales y la integración en las cadenas de proveedores a empresas de diferentes países, algunos en vías de desarrollo, que podrían verse beneficiados por la extensión de prácticas de RSE desde empresas europeas o empresas radicadas en Europa.

La Unión Europea se reconoce pionera en su trabajo sobre la RSE, pues se trata de la única instancia de carácter supranacional que lo ha abordado hasta el momento, aunque manifiesta que se trata de un trabajo complejo y progresivo, dada la disparidad de acciones de RSE que coexisten en la actualidad. Por ello se recuerda la necesidad de cohesión y puesta en común de buenas prácticas e iniciativas novedosas, así como la importancia del intercambio de ideas y alianza entre las partes empresariales, sociales y políticas. En este sentido, la administración pública debe adoptar una función coordinadora e impulsora de estas iniciativas, desarrollando a su vez la RSE en sus administraciones públicas e incentivando su desarrollo en las empresas locales. El trabajo desarrollado en el contexto comunitario es fundamental como marco para la formulación de políticas nacionales de RSE pues, al mismo tiempo, mediante el avance en políticas de RSE nacionales y a través de la cooperación entre Estados miembros, también se puede contribuir a una mejor definición de una política europea común basada en los fundamentos 
del desarrollo sostenible. Estos postulados centrados desde sus inicios en el desarrollo de una estrategia comunitaria de RSE capaz de fortalecer el modelo social europeo, han abierto el debate sobre la importancia de extender las prácticas de RSE más allá de los límites geográficos de la Europea comunitaria, pues progresivamente se ha ido valorando el papel activo de las empresas en todas las fases y lugares de su cadena productiva y de distribución. La ampliación del campo de visión y actuación es coherente con el desarrollo de los mercados globales y, desde la actual crisis económica internacional, podríamos considerar que se trata además de una ampliación imprescindible. No en vano de los cuatro grupos de trabajo constituidos en la primera reunión del Consejo Estatal de Responsabilidad Social de las Empresas, uno estará centrado en el análisis del papel de la RSE en el contexto de la crisis económica.

Tal como se indica en la Resolución del Parlamento Europeo sobre la responsabilidad social de las empresas: una nueva asociación, de 13 de marzo de 2007, una mayor responsabilidad social y medioambiental de las empresas puede responder a los nuevos retos sociales vinculados a la globalización económica. Pero como se recoge en esta Resolución, es necesario diferenciar qué se entiende por una adecuada estrategia de RSE y cuáles son los criterios para definir una buena práctica en la materia. Este trabajo es necesario en un momento como el actual en el que la RSE se considera en muchas ocasiones una "etiqueta" bajo la que pueden englobarse acciones diversas y dispersas no siempre relacionadas con una estrategia de empresa socialmente responsable. Para ello será adecuado revisar el propio concepto de voluntariedad de las empresas en materia de responsabilidad social y valorar la importancia de ofrecer criterios objetivos para evaluar las buenas prácticas y, si cabe, incentivarlas desde las administraciones públicas. Esto también debería hacer referencia a todas las organizaciones, pues si bien en repetidas ocasiones la doctrina comunitaria recuerda que se trata de un concepto válido y necesario para cualquier empresa con independencia de su tamaño, no resulta tan explícita la validez para entidades no empresariales. En este sentido defendemos la importancia de aplicar modelos de gestión socialmente responsable en cualquier organización, con independencia tanto de su tamaño como de su sector de actividad, entidad jurídica (pública o privada) y finalidad (lucrativa o no lucrativa).

En último lugar se considera oportuno plantear una reflexión sobre la escasa visibilidad del ámbito académico en el debate comunitario y estatal sobre la RSE. Diferentes áreas de conocimiento (en especial la Sociología y la Economía) podrían realizar importantes aportaciones en tres niveles de aproximación a la responsabilidad social corporativa: teórico, aplicado e institucional. En este sentido, conviene recordar 
que el Pacto Mundial de Naciones Unidas (Global Compact), entre los principios para una formación directiva responsable, incluye el desarrollo de investigación conceptual y empírica que ayude a comprender la función, dinámica e impacto de las organizaciones en la creación de sostenibilidad social, medioambiental y valor económico. Como ejemplo en nuestro país podemos comprobar cómo la presencia académica tanto en el Foro de Expertos en RSE (que recoge entre sus recomendaciones la promoción del tema en las enseñanzas universitarias) como en el Consejo Estatal de RSE podría calificarse de anecdótica, pues entre los 56 vocales y otros tantos suplentes en representación de organizaciones empresariales, sindicales, políticas, ONG y entidades de reconocido interés en el ámbito de la RSE, solamente dos vocales y dos suplentes participan en calidad de "expertos académicos", lo que supone un circunstancial $3,57 \%$ del total de vocales.

\section{ANEXO I}

\begin{tabular}{|c|l|l|}
\hline Año & Iniciativa comunitaria & \multicolumn{1}{|c|}{ Elemento que introduce a debate } \\
\hline 2000 & Estrategia de Lisboa & $\begin{array}{l}\text { Convertir Europa en la economía basada en el } \\
\text { conocimiento más competitiva y dinámica del } \\
\text { mundo. Crecimiento económico sostenible y con } \\
\text { mayor cohesión social. }\end{array}$ \\
\hline 2000 & Consejo de Niza & $\begin{array}{l}\text { Reforzar la política social frente a los nuevos } \\
\text { desafíos de la sociedad del conocimiento. }\end{array}$ \\
\hline 2001 & $\begin{array}{l}\text { Consejo de } \\
\text { Gotemburgo }\end{array}$ & $\begin{array}{l}\text { Crecimiento económico respetuoso con los recur- } \\
\text { sos naturales. Desarrollo sostenible. }\end{array}$ \\
\hline 2001 & $\begin{array}{l}\text { Resolución relativa al } \\
\text { seguimiento del Libro sobre la } \\
\text { Verde sobre la RSE }\end{array}$ & $\begin{array}{l}\text { Fomento de un marco europeo para la res- } \\
\text { ponsabilidad social de las empresas. Apoyo al } \\
\text { carácter voluntario de la RSE. Reconocimiento } \\
\text { de la importancia de integración en el contexto } \\
\text { internacional de las políticasy prácticas europeas } \\
\text { en la materia. }\end{array}$ \\
$\begin{array}{l}\text { Reconocimiento de la capacidad de la RSE para } \\
\text { fomentar un alto nivel de cohesión social, protec- } \\
\text { ción medioambiental y respeto de los derechos } \\
\text { fundamentales. Valoración de la investigación, } \\
\text { difusión de buenas prácticas y formación de } \\
\text { personal directivo sobre la materia. }\end{array}$ \\
\hline
\end{tabular}




\begin{tabular}{|l|l|l|}
\hline 2002 & $\begin{array}{l}\text { Foro multilateral euro- } \\
\text { peo sobre RSE }\end{array}$ & $\begin{array}{l}\text { Necesidad de incluir los puntos de vista de todos } \\
\text { los agentes interesados a escala europea, nacio- } \\
\text { nal, regional y local. Valoración de la RSE como } \\
\text { práctica estratégica de las empresas relacionada } \\
\text { con sus operaciones comerciales y con las rela- } \\
\text { ciones con sus públicos objetivo. } \\
\text { Superación de la visión filantrópica para } \\
\text { incluir la RSE en el marco estratégico de las } \\
\text { organizaciones. }\end{array}$ \\
\hline 2003 & $\begin{array}{l}\text { Resolución del Consejo } \\
\text { de UE relativa a la res- } \\
\text { ponsabilidad social de } \\
\text { las empresas }\end{array}$ & $\begin{array}{l}\text { Importancia de la trabajar de forma paralela al } \\
\text { desarrollo de una estrategia comunitaria en todos } \\
\text { los Estados miembros. }\end{array}$ \\
\hline 2005 & $\begin{array}{l}\text { Plataforma de Acción } \\
\text { recogida en la Comuni- } \\
\text { cación de la Comisión } \\
\text { relativa a la revisión de } \\
\text { la Estrategia para un } \\
\text { desarrollo sostenible }\end{array}$ & $\begin{array}{l}\text { Inclusión de la responsabilidad social de las } \\
\text { empresas y de los interlocutores sociales dentro } \\
\text { de los principios rectores de las políticas para un } \\
\text { desarrollo sostenible. }\end{array}$ \\
\hline 2006 & $\begin{array}{l}\text { Comunicación "Hacer } \\
\text { de Europa un polo de } \\
\text { excelencia de la Res- } \\
\text { ponsabilidad Social de } \\
\text { las Empresas" }\end{array}$ & $\begin{array}{l}\text { Necesidad de fijar límites al protagonismo de la } \\
\text { economía de mercado en la escena global. Im- } \\
\text { portancia de la movilización a favor del bienestar } \\
\text { social en las democracias modernas, valorando } \\
\text { el papel activo de las empresas. }\end{array}$ \\
\hline 2006 & $\begin{array}{l}\text { Alianza Europea para } \\
\text { la RSE }\end{array}$ & $\begin{array}{l}\text { Importancia del compromiso de las empresas } \\
\text { con el desarrollo de los valores y principios } \\
\text { europeos. Confianza en la capacidad de la RSE } \\
\text { para aunar sostenibilidad y competitividad en } \\
\text { los mercados. }\end{array}$ \\
\hline 2007 & $\begin{array}{l}\text { Resolución "sobre la } \\
\text { responsabilidad social } \\
\text { de las empresas: una } \\
\text { nueva asociación". }\end{array}$ & $\begin{array}{l}\text { Impacto de la RSE en las cadenas mundiales de } \\
\text { proveedores. Necesidad de actuar con respon- } \\
\text { sabilidad social en todos los niveles y contextos } \\
\text { de la cadena de producción y consumo (no sólo } \\
\text { en el ámbito local comunitario). Necesidad de } \\
\text { establecer criterios sobre buenas prácticas en } \\
\text { materia de RSE. }\end{array}$ \\
\hline
\end{tabular}




\section{Bibliografía}

Comisión de las Comunidades Europeas (2001), Libro Verde. Fomentar un marco europeo para la responsabilidad social de las empresas, Bruselas, Comisión de las Comunidades Europeas.

ARAQUe, R. (2002), "Una aportación al debate sobre el Libro Verde de la responsabilidad social de la empresa", Revista de Fomento Social, n. ${ }^{\circ} 228$, pp. 595-628.

- (2004), "Factores condicionantes de la responsabilidad social de la empresa: los límites de lo posible", Revista de Fomento Social, n. ${ }^{\circ} 235$, pp. 553-592.

CAMACHO, I. (2004), "La responsabilidad social de la empresa: un proceso abierto", Revista de Fomento Social, n. ${ }^{\circ}$ 233, pp. 75-98.

MeADOWs, D., RANDERS, J. y MeADows, D. (2006), Los límites del crecimiento 30 años después, Barcelona, Galaxia Gutenberg.

PARDO, M. (1997), "El Desarrollo", en J. Ballesteros y J. Pérez Adán, Sociedad y medio ambiente, Madrid, Trotta, pp. 187-205.

Real Decreto 221/2008, de 15 de febrero, por el que se crea y regula el Consejo Estatal de Responsabilidad Social de las Empresas, BOE núm. 52, viernes 29 de febrero de 2008.

Recomendación 2005/601/CE DEL CONSEJO, de 12 de julio de 2005, relativa a las orientaciones generales de las políticas económicas de los Estados miembros y la Comunidad (2005-2008), Diario Oficial L 205 de 6.8.2005.

Resolución del Consejo de 6 de febrero de 2003 relativa a la responsabilidad social de las empresas (2003/C 39/02), Diario Oficial de la Unión Europea C $39 / 4,18.2 .2003$.

Resolución del Consejo de la Unión Europea relativa al seguimiento del Libro Verde sobre la responsabilidad social de las empresas (2002/C 86/03), Diario Oficial de las Comunidades Europeas, C86, 10.4.2002.

Rodríguez, M. A. (2007), "La Responsabilidad Social de la Empresa", en A. Olcese (Dir.), La Responsabilidad Social de la Empresa (RSE). Propuesta para una nueva economía de la empresa responsable y sostenible, Madrid, Real Academia de Ciencias Económicas y Financieras, pp. 89-119 
SAGARDOY, I. (2007), "La dimensión de la RSE en la Unión Europea", en A. Olcese (Coord.), La Responsabilidad Social de la Empresa (RSE). Propuesta para una nueva economía de la empresa responsable y sostenible, Madrid, Real Academia de las Ciencias Económicas y Financieras, pp. 135-150.

Savitz, A. W. y Weber, K. (2006), The Triple Bottom Line, San Francisco, Jossey-Bass.

VAN LANCKER, A. (2001), La Agenda Social Europea: La ambición social de Europa, Foro de Seguridad Social, n 5. <http://www.foross.org/revista05/a04.php> [Consulta: 3 julio 2008].

\section{Documentos de acceso electrónico}

AnNan, K. Discurso de lanzamiento de Global Compact. <http://www.pactomundial.org/recursos/doc/Global_Compact/Politicas_y_documento_Global_Compact/49455_1391392007164051.pdf> [Consulta: 10 octubre 2007].

Comunicación al Consejo Europeo de Primavera: Trabajando juntos por el crecimiento y el empleo. Relanzamiento de la Estrategia de Lisboa. COM (2005) 24 final. < http://eur-lex.europa.eu/es/index.htm> [Consulta: 10 julio 2008].

Comunicación de la Comisión al Parlamento Europeo, al Consejo y al Comité Económico y Social Europeo, 22 de marzo de 2006. "Poner en práctica la asociación para el crecimiento y el empleo: Hacer de Europa un polo de excelencia de la Responsabilidad Social de las Empresas". <http://eur-lex.europa.eu/es/index. $\mathrm{htm}>$ [Consulta: 4 julio 2008].

Comunicación de la Comisión relativa a la racionalización de los ciclos anuales de coordinación de la política económica y de la política de empleo (COM (2002) 487). <http://eur-lex.europa.eu/es/index.htm> [Consulta: 22 junio 2008].

Comunicación de la Comisión relativa a la responsabilidad social de las empresas: una contribución empresarial al desarrollo sostenible (COM (2002) 347 final, no publicada en el D.O.) <http://eur-lex.europa.eu/es/index.htm> [Consulta: 2 julio 2008].

Comunicación de la Comisión relativa a la revisión de la Estrategia para un desarrollo sostenible. Plataforma de acción. <http://www.hablamosdeeuropa.es> [Consulta: 1 julio 2008]. 
Consejo Europeo de Gotemburgo. Conclusiones de la Presidencia. <http://www. hablamosdeeuropa.es> [Consulta: 30 junio 2008].

Consejo Europeo de Lisboa, 23 y 24 de marzo 2000. Conclusiones de la Presidencia. <http://www.europarl.europa.eu/summits/lis1_es.htm> [Consulta: 19 junio 2008].

Desarrollo sostenible en Europea para un mundo mejor: Estrategia de la UE para un desarrollo sostenible. Comunicación de la Comisión Europea. < http://eur-lex. europa.eu/es/index.htm> [Consulta: 30 junio 2008].

Estrategia a favor del desarrollo sostenible. <http://europa.eu/scadplus/leg/es/ lvb/l28117.htm> [Consulta: 10 julio 2008].

Estrategia de Lisboa. <http://europa.eu/scadplus/glossary/lisbon_strategy_ es.htm> [Consulta: 17 junio 2008].

Global Compact, Responsabilidad cívica corporativa: definición de las nuevas responsabilidades. <http://www.pactomundial.org> [Consulta: 24 octubre 2007].

Global Reporting Initiative, Guía para la elaboración de memorias de sostenbilidad. Versión G-3 (2000-2006). <http://www.globalreporting.org> [Consulta: 25 mayo 2008].

La Agenda Social 2005-2010. Una Europa social en la economía global. Empleo y oportunidades para todos. <http://ec.europa.eu/employment_social/publications> [Consulta: 8 julio 2008].

Líneas Directrices de la OCDE para empresas multinacionales. <http://www.oecd. org/dataoecd/21/20/16975360.pdfs [Consulta: 8 julio 2008].

Informe del Foro de Expertos en Responsabilidad Social de las Empresas del Ministerio de Trabajo y Asuntos Sociales. <http://www.mtas.es/Empleo/economia-soc/ Default.htm> [Consulta: 12 julio 2008].

Orientaciones generales de política económica (2005-2008). <http://europa.eu/ scadplus/leg/es/lvb/l25078.htm> [Consulta: 21 junio 2008].

Pacto de estabilidad y coordinación de las políticas económicas. <http://europa. eu/scadplus/leg/es/s01040.htm> [Consulta: 22 junio 2008]. 
Resolución del Parlamento Europeo sobre la Comunicación de la Comisión relativa a la responsabilidad social de la empresas: una contribución empresarial al desarrollo sostenible (COM(2002) 347). < http://eur-lex.europa.eu/es/index. $\mathrm{htm}>$ [Consulta: 9 julio 2008].

Resolución del Parlamento Europeo, de 13 de marzo de 2007, sobre la responsabilidad social de las empresas: una nueva asociación. <http://www. europarl.europa.eu/sides/getDoc.do?pubRef=-//EP//TEXT+TA+P6-TA-20070062+0+DOC+XML+VO//ES> [Consulta: 11 julio 2008].

Responsabilidad Social de las Empresas: una contribución empresarial al desarrollo sostenible. <http://europa.eu/scadplus/leg/es/lvb/n26034.htm> [Consulta: 1 julio 2008].

Sexto Programa de Acción de la Comunidad Europea en materia de Medio Ambiente. <http://europa.eu/scadplus/leg/es/lvb/l28027.htm> [Consulta: 30 junio 2008].

Social Accountability 8000. < http://www.sa-intl.org/> [Consulta: 9 julio 2008]. 